\title{
A case-control study of medical, psychological and socio- economic factors influencing the severity of chronic rhinosinusitis*
}

\author{
Carl Philpott', Sally Erskine 1,2, Claire Hopkins³, Emma Coombes ${ }^{1}$, \\ Naveed Kara ${ }^{4}$, Vishnu Sunkareneni ${ }^{5}$, Shahram Anari ${ }^{6}$, Mahmoud Salam7, \\ Amir Farboud ${ }^{8}$, Allan Clark', on behalf of the CRES Group \\ 'Norwich Medical School, University of East Anglia, Norwich, UK \\ 2 ENT Department, James Paget University Hospital NHS Foundation Trust, Gorleston, UK \\ ${ }^{3}$ ENT Department, Guy's and St Thomas' NHS Foundation Trust, London, UK \\ ${ }^{4}$ ENT Department, Darlington Memorial Hospitals NHS Foundation Trust, Darlington, UK \\ ENT Department, Royal Surrey County Hospital, Surrey, UK \\ ${ }^{6}$ ENT Department, Heart of England NHS Foundation Trust, Birmingham, UK \\ ENT Department, Ipswich Hospital NHS Trust, Ipswich, UK \\ ${ }^{3}$ ENT Department, Betsi Cadwaladr University Health Board Wrexham Maelor Hospital, Wrexham, UK
}

Rhinology 54: 134-140, 2016 DOl:10.4193/Rhino15.272

*Received for publication: August 31, 2015 Accepted: December 12, 2015

This study is reported according to

the STROBE statement for observational studies.

\begin{abstract}
Background: Chronic rhinosinusitis (CRS) is a common and debilitating disorder. Little is known about the epidemiology of this disease. The aims of the study were to identify differences in socio-economic variables and quality of life between patients with chronic rhinosinusitis and healthy controls, to identify any significant associations between CRS and other medical co-morbidities, psychiatric disease or environmental exposure and to explore the experience of CRS from the perspective of CRS sufferers.
\end{abstract}

Methods: Participants were recruited from ENT clinics from 30 centres across the UK. They completed a study-specific questionnaire considering environmental, medical and socio-economic factors, and SF-36 and SNOT-22 scores. All participants with CRS were diagnosed by a clinician and categorised as having CRS (with polyposis, without polyposis or allergic fungal rhinosinusitis (AFRS)). Controls included family and friends of those attending ENT outpatient clinics and hospital staff who had no diagnosis of nose or sinus problems and had not been admitted to hospital in the previous 12 months.

Results: A total of 1470 study participants (1249 patients and 221 controls) were included in the final analysis. Highly significant differences were seen in generic and disease-specific quality of life scores between CRS sufferers and controls; mean SNOT-22 score 45.0 for CRS compared with 12.1 amongst controls. There were no clear differences in socioeconomic variables including social class, index of multiple deprivation and educational attainment between cases and controls. Common comorbidities with a clear association included respiratory and psychiatric disorders, with a higher frequency of reported upper respiratory tract infections.

Conclusions: CRS is associated with significant impairment in quality of life and with certain medical co-morbidities. In contrast to other common ENT disorders, no socioeconomic differences were found between patients and controls in this study.

Key words: chronic rhinosinusitis, health inequalities, quality of life, respiratory disease, socioeconomic factors

\section{Introduction}

Chronic rhinosinusitis (CRS) affects a significant proportion of the population; a recent European study found a prevalence of
$11 \%{ }^{(1)}$. Despite this, the epidemiology of CRS and in particular its association with socioeconomic variables has not been extensively explored. The European Position Paper on Rhinosinu- 
sitis and Nasal Polyps (EPOS 2012) has stated under the heading 'Research Needs' that studies are required to consider 'the prevalence of and predisposing factors for CRSsNP and CRSwNP' and to 'investigate the impact of psychological problems such as depression, stress exposure and anxiety on subjective severity' (2). A previous study of 158 patients has suggested significant morbidity in CRS, with quality of life scores worse than amongst those with other chronic diseases such as lower back pain ${ }^{(3)}$. This significant effect on an individual's functioning and productivity, has an impact upon workforce productivity, since CRS primarily affects those aged $40-60$ years. CRS has been identified as one of the top ten most costly diseases for US employers ${ }^{(4)}$. Despite its high prevalence and impact, the pathophysiology and hence optimal treatment for CRS are not well understood, but it is thought to be a spectrum of diseases with different underlying aetiologies and pathological features. Infection (viral, bacterial and fungal) and underlying genetic tendencies may all be contributory factors. CRS is currently subdivided into two main types - CRS with and without nasal polyps (CRSwNP and CRSsNP, respectively), as exemplified by EPOS2012 ${ }^{(2)}$ to reflect coarsely differing gross pathophysiology (eosinophilic or neutrophilic) but allergic fungal rhinosinusitis (AFRS) is an increasingly recognised distinct subtype of CRSwNP.

Deprivation is known to be associated with increasing morbidity and mortality, and is therefore important to consider in understanding the epidemiology of any disease, since it is a potentially reversible determinant of health ${ }^{(5)}$. Many reasons for this relationship have been explored. Poor nutrition leads to poor mental and physical development. Cold or damp housing is associated with increased risk of respiratory diseases, and overcrowded housing is associated with infectious diseases ${ }^{(6)}$. Behavioural differences which may be related to lack of resources or poor education also contribute to socioeconomic variation in health, with smoking being the most common example. Reduced access to health care, genetic factors and adverse social conditions also contribute ${ }^{(7)}$. Within otorhinolaryngology it is known that one of the most common risk factors for otitis media is socioeconomic status ${ }^{(8)}$, with more deprived children more likely to suffer adversely with the condition. There is controversy as to the role of deprivation in other upper respiratory problems; the direction of association between asthma and socioeconomic status varies widely between studies ${ }^{(9,10)}$.

By developing our understanding about the socio-economic and co-morbidity factors that may influence CRS, specific comorbid associations and high-risk population groups might be identified. This information could enable health practitioners, including ENT specialists and General Practitioners, to better tailor management to individual patients' needs. Epidemiological studies outwith Europe have shown varying prevalence rates. In Canada, the prevalence of CRS, defined as an confirmatory answer to the question, 'Has the patient had sinusitis diagnosed by a health professional lasting for more than 6 months?' ranged from $3.4 \%$ in male to $5.7 \%$ in female subjects ${ }^{(11)}$. In Korea, the overall prevalence of CRS, defined as the presence of at least 3 nasal symptoms persisting for more than 3 months, together with an endoscopic finding of nasal polyps and/or mucopurulent discharge within the middle meatus, was $1.01 \%{ }^{(12)}$. A comparative study between the north of Scotland and the Caribbean found that in ENT clinics across both countries, there was a similar prevalence of CRS (9.6\% and 9.3\%, respectively) ${ }^{(13)}$.

To date, no large scale study into the epidemiology of CRS has been undertaken in the UK, and the Chronic Rhinosinusitis Epidemiology Study (CRES) meets this need. The primary aim of the study was to identify differences in socio-economic variables and quality of life between patients with chronic rhinosinusitis and healthy controls. Secondary aims were to identify any significant associations between CRS and other medical co-morbidities, psychiatric disease or environmental exposure and to explore the experience of CRS from the perspective of CRS sufferers.

\section{Materials and methods}

\section{Study design and setting}

CRES was approved by the Oxford C Research Ethics Committee, sponsored by the University of East Anglia (UEA) and funded by the Anthony Long and Bernice Bibby Trusts. The study was conducted as a prospective case-control questionnaire study. Following a pilot study of the questionnaire in 2006, the study commenced recruitment in ENT departments of the East Anglia region (East of England Deanery) of the UK in 2007. Following elevation to the National Institute of Health Research Clinical Research Network Portfolio in 2012, a total of 30 sites from around the UK (including the devolved nations of Wales and Scotland) joined the study which ran between 2007 and 2013. The study specific questionnaire was anonymous and therefore consent was implied through participation. Participant information leaflets were provided. Questionnaires were completed on one occasion only either before leaving the clinic or taken home and returned by post in Freepost envelopes. A qualitative arm of the study was undertaken in 2012. This is published elsewhere ${ }^{(14,15)}$.

\section{Participants}

The diagnosis of CRS was confirmed by an Otorhinolaryngologist. CRS patients presenting to secondary care ENT outpatient clinics were invited to participate in the study, regardless of symptom or disease severity or previous treatment, provided they conformed to the following criteria:

\section{Inclusion criteria:}

CRS with or without polyps as defined by the criteria laid out in EPOS $2012^{(2)}$. Symptoms must be present for at least 12 weeks 
and include:

- Nasal blockage/obstruction/congestion and/or nasal discharge (anterior/posterior nasal drip) and

- Either facial pain/pressure and/or reduction or loss of smell and additionally:

- Endoscopic signs of: polyps and/or mucopurulent discharge primarily from middle meatus and/or; oedema/mucosal obstruction primarily in middle meatus and/or

- CT changes: mucosal changes within the ostiomeatal complex and/or sinuses

Any patients with nasal polyps placed in the AFRS category met the Bent and Kuhn criteria ${ }^{(16)}$ or the St Paul's Sinus Centre modification of this ${ }^{(17)}$.

Patients and controls included were all adult.

\section{Exclusion criteria:}

- Patients unable to comprehend written English.

For the control group:

- $\quad$ Patients with active sinonasal disease - e.g. acute or chronic forms of rhinitis/rhinosinusitis (as determined by patient history or SNOT-22 score of 10 or more

- No chronic medical conditions being actively treated or

- hospitalisation within the last 12 months

Controls included family and friends of those attending ENT outpatient clinics and hospital staff.

\section{Variables and data sources}

The study questionnaire was designed with the input of the East of England Research Design Service and included study specific questions relating to socio-economic, environmental and medical co-morbid variables as well as the validated Short Form 36 Quality of Life (QoL) measure (SF-36) ${ }^{(18)}$ measure and the Sino-Nasal Outcome Test questionnaire (SNOT-22) ${ }^{(19)}$.

\section{Socioeconomic variables}

Respondents were asked to enter data for occupation, highest academic qualification, rural/urban location, duration of residency, proximity to crops, postcode, annual income, ethnicity and household occupancy. Social class based on the National Statistics Socio-economic Classification (NS-SEC) ${ }^{(20)}$ and the Index of Multiple Deprivation ${ }^{(21)}$ were calculated and used to assess socio-economic differences. Participants were also asked about tobacco and alcohol consumption.

\section{Medical co-morbidities}

Data requested under this category included information on psychiatric disorders, frequency of common respiratory illnesses, past medical and surgical history, drug history, known and suspected allergies and sensitivities to aspirin and foods high in salicylate content.

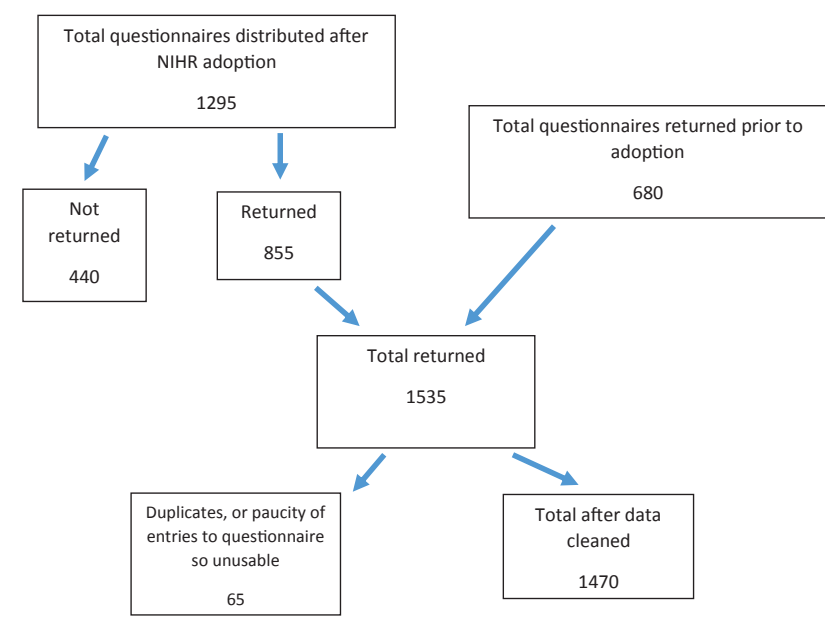

Figure 1. Participant flow.

\section{Sample size calculation}

The purpose of the study was to look for common associations between CRS, and primarily social class (as determined by occupation, highest qualification and household income), and CRS and housing status (as determined by occupancy of household in conjunction with social class). These two factors have been used to determine the size of the study sample required. For socio-economic scores, the standard approach is to compare the proportion of subjects in the lower social classes to everyone else. In order for the study to have $80 \%$ power to detect a difference of $10 \%$ in "low social class" between controls and CRS patients, assuming a $30 \%$ rate in the CRS patients, with approximately 5 CRS patients to 1 control patient, 965 CRS patients and 193 controls were required.

For the purposes of assessing QoL, assuming that a change in QoL of 10 units on SF-36 can be shown (standard deviation of 20 ), then to have $80 \%$ power to detect this difference (at the $5 \%$ level of significance), 38 controls and 190 cases would be needed. This would need to be increased by $20 \%$ to allow for the non-normality of QoL and the study would need 46 controls and 228 cases.

\section{Results}

\section{Participants}

A total of 1470 participants with an age range of 17-102 years were recruited. Following adoption on to the NIHR portfolio, recruitment rates improved to a peak of 120 subjects per month. After adoption, the overall recruitment was $66 \%$ of those invited to participate. Participants who were recruited prior to adoption onto the portfolio make up the additional participants. Information on recruitment rates prior to adoption was not collected and there is no information on reasons for non-participation. A total of 1535 questionnaires were returned, reduced to 1470 
Table 1. Demographic information for each of the included subgroups.

\begin{tabular}{ccccc|}
\hline & Controls & CRSsNP & CRSwNP & AFRS \\
\hline Participants & 221 & 553 & 651 & 45 \\
& 143 & 259 & 185 & 19 \\
Females & $(68.4 \%)$ & $(53.1 \%)$ & $(32.2 \%)$ & $(43.2 \%)$ \\
& 47.3 & 51.8 & 56.0 & 56.1 \\
Mean Age (SD) & $(14.9)$ & $(15.3)$ & $(14.6)$ & $(12.7)$ \\
\hline Range & $19-82$ & $18-84$ & $17-102$ & $20-76$ \\
\hline
\end{tabular}

eligible after checking for duplicates and missing information. See Figure 1 for participant flow.

\section{Descriptive and outcome data}

The 1470 participants included 709 males and 606 females (155 undeclared); $44 \%$ had CRSsNP and $56 \%$ had CRSwNP or AFRS. As demonstrated in Figure 2, the geographic distribution of study participants includes a wide range of rural and urban areas of the country and in 3 out of the 4 devolved nations. Table 1 shows detailed demographic information for each of the included subgroups. The full amount of data available was used for each relevant analysis; for example, if SNOT-22 was completed but not SF-36, participants were included in SNOT-22 analysis but not SF-36. Similarly, for all socioeconomic factors all participants who completed the relevant question were included in that particular analysis, to maximize use of the available data.

\section{Main results}

\section{Socio-economic outcomes}

Social class is an individual-level assessment based on selfreported occupation: 1350 respondents (91.8\%) provided this information. Due to the small number of individuals in some categories, classes 1.1 and 1.2 were combined, 4 and 5 were combined, and 7 and 8 were combined to assess differences. There was a significant association between social class and CRS status ( $p=0.002$ ); however when adjusted for age and sex the difference was no longer statistical significant $(p=0.0684)$ and there was no specific direction of association.

The index of multiple deprivation (IMD) was also calculated as a measure of socioeconomic status (21). This is an area-based deprivation measure based on postcode. IMD scores for each postcode are based on government statistics measuring relative levels of deprivation in small areas of England called Lower Layer Super Output Areas (LSOAs). Domains include income, employment, health and disability, education, skills and training, barriers to housing and services, living environment and crime. Most of the indicators used in these statistics are from 2008. There were no significant differences between those with CRS and controls ( $p=0.115$ ); nor did any appear after adjusting for age and sex (mean difference $-1.36,95 \% \mathrm{Cl}$ : -3.00 to $0.29, \mathrm{p}=0.107$ ).
Table 2. Quality of life and socioeconomic variables.

\begin{tabular}{|lcc|}
\hline & $\begin{array}{c}\text { NOT-22 (Spearman rho, } \\
\text { p-value) }\end{array}$ & $\begin{array}{c}\text { SF-36 (Spearman rho, } \\
\text { p-value) }\end{array}$ \\
\hline Social Class & $0.0935 ; p=0.001$ & $-0.1545 ; \mathrm{p}<0.0001$ \\
\hline IMD & $-0.0131 ; \mathrm{p}=0.6449$ & $-0.0035 ; \mathrm{p}=0.9006$ \\
\hline $\begin{array}{l}\text { Household } \\
\text { occupancy }\end{array}$ & $0.0017 ; \mathrm{p}=0.9518$ & $0.0695 ; \mathrm{p}=0.0092$ \\
\hline $\begin{array}{l}\text { Educational } \\
\text { attainment }\end{array}$ & $-0.0473 ; \mathrm{p}=0.1359$ & $0.1010 ; \mathrm{p}=0.0012$ \\
\hline
\end{tabular}

The number of occupants in the household of the participant was also considered; households of controls tended to have more occupants than households of those with CRS $(p=0.003)$, however this was not significant after adjusting for age and sex $(p=0.275)$. Household income (according to the participant) was intended to be used as a further socioeconomic measure but only two thirds of respondents provided information although no significant differences were found. Mean income was $£ 41,118.63$ for controls and $£ 42,800.02$ for those with CRS. This highest educational qualification achieved by the participant showed no significant differences between controls and those with CRS ( $p=0.599$ ).

\section{Quality of Life}

Quality of life was measured using the SF-36 and SNOT-22. There was a statistically significant association between SNOT-22 and social class, but only a weak correlation was detected (Spearman rho $=0.0935, p=0.001)$. There was no correlation between SNOT22 score and IMD, number of household occupants or educational attainment. There were statistically significant associations between three socioeconomic variables and SF-36 but all correlations were weak. Results are shown in table 2. There were significant differences in mean scores between controls and those with CRS for both the SF-36 and SNOT-22, before and after adjustment for age and gender differences. Controls had better scores for both scales as illustrated in table 3. A further detailed analysis of the SNOT-22 subscales and differences between CRS subtypes will be reported separately.

\section{Co-morbidities}

Several co-morbidities were higher amongst those with CRS than controls, including psychiatric problems $(p=0.001)$ and respiratory issues.

\section{CRS and mood disturbances}

Chi-squared test showed significant differences between participant subgroups for both depression $p=0.03$ and anxiety $p=0.04$ and between mental health domain scores on SF-36 $(p=0.05)$. This will be published in detail at a later date. 
Table 3. Quality of life and diagnosis.

\begin{tabular}{|lccccccc} 
& CRS & Controls & Unadjusted & p-value & Age and gender adjusted & p-value \\
\hline SF-36 & $67.8(20.5)$ & $80.8(15.1)$ & $-12.97(-15.81,-10.12)$ & $<0.001$ & $-14.32(-17.34,-11.30)$ & $<0.001$ \\
\hline SNOT-22 & $45.0(21.4)$ & $12.1(13.9)$ & $32.85(29.78,35.92)$ & $<0.001$ & $36.40(33.16,39.64)$ & $<0.001$ \\
\hline
\end{tabular}

\section{Allergies}

Those with CRS were more likely to report respiratory tract sensitivity to aspirin $(p=0.003)$, wine $(p<0.001)$, fruits $(0.034)$ and nuts (0.026), but not to spicy food, drinks or vegetables. Further analysis is required for the free text answers regarding inhalant allergies and will be reported elsewhere.

\section{Respiratory}

Asthma had a strong association with CRS $(<0.001)$ with those in the AFRS subgroup most frequently affected. Those with CRS were more likely to report suffering from upper respiratory tract infections (URTIs) 'often' OR=7.39 (95\% Confidence interval [CI]: 3.31-16.51) or 'frequently' 30.25 (95\% Cl 9.77, 93.63).

\section{Burden of surgery}

Amongst all CRS patients, $45 \%$ had undergone some form of sinonasal surgery previously (defined as one or more of polypectomy, endoscopic sinus surgery (ESS), septoplasty, turbinate surgery, rhinoplasty) including 325 (26\%) who had received at least one nasal polypectomy and 169 (14\%) who had undergone at least one instance of ESS (separately or concurrently). The mean number of previous surgeries per patient in those had undergone multiple procedures was 3.3 (range 2-30) and a mean duration of time of 10 years since the last procedure. A detailed analysis of the surgical data is reported elsewhere (22).

\section{Lifestyle and environmental exposure}

There were no significant differences in smoking or alcohol consumption between controls and those with CRS. Nor were there significant differences in proportions of those living near crops between those with CRS and controls. Data on air pollution for all recruitment sites is currently being sought and will be reported separately.

\section{Discussion}

Key results

Sufficient data on socioeconomic status were collected to enable the primary objectives to be determined. There were no significant differences in socio-economic variables as measured by social class, IMD or household occupancy between those with and without CRS. There have been few previous studies investigating the association between CRS and different measures of socioeconomic status, particularly in the UK. A similar sized epidemiological study of residents of Sao Paulo also found no statistically significant differences in CRS prevalence according to number of household residents, educational achievement or income of head of household, but did find a significant association between presence of CRS and belonging to a low-income group ${ }^{(23)}$, although it is noted that social structure in Sao Paulo is different to the UK. Another study of 127 patients found that lower family income was related to worse self-reported sinus disease (although there was no difference in objective sinus disease based on Lund-Mackay score) (24). A study considering markers of disease severity amongst 93 patients with AFRS in North Carolina, found that bone erosion and orbitocranial involvement were associated with lower income, rural counties, poor housing quality, and less health care access ${ }^{(25)}$. Some studies have found that comparable chronic diseases such as asthma have a strong association between poverty and disease severity ${ }^{(10)}$ but this is controversial ${ }^{(9)}$. Our study found no differences in education attainment between cases and controls.

There were weak but statistically significant associations between SNOT-22 score and social class, and SF-36 score and social class, household occupancy and educational attainment. Although there is sparse literature investigating such associations amongst those with CRS, Kilty et al found that those with a lower educational level scored more highly on a sinus symptom score ${ }^{(24)}$

Highly significant differences were seen in generic and diseasespecific QoL scores between cases and controls, with cases having less favourable scores on both SF-36 and SNOT-22, emphasising that CRS patients have a significant impairment of their QoL. This is supported by several previous studies ${ }^{(26)}$. Potential explanations for the association between socioeconomic variables and disease severity are likely to be multi-factorial, reflecting the wide range of influencing aetiological factors in CRS as well as individuals' perceptions of symptoms. Several comorbidities were significantly more likely to be found amongst those with CRS than controls, including psychiatric problems including mood disturbances and asthma. Studies considering the biopathophysiological mechanisms which could be involved in the association between socioeconomic status and the development of asthma have proposed family stress and endotoxin exposure in low-income households as a factor in development and experience of symptoms ${ }^{\left({ }^{(10)}\right.}$. For example, caregiver stress in early life has been associated with increased levels of TNF- $\alpha$ 
in infants, which is known to be a pro-inflammatory cytokine in asthma ${ }^{(27)}$.

The proportion of those reporting allergies including aspirin, wine, fruits and nuts was higher amongst cases than controls. This is supported by several previous studies ${ }^{(28)}$. There were no significant differences in smoking habits or alcohol intake between cases and controls. Existing literature varies as to the nature of any association with CRS. Despite being known to reduce mucociliary clearance time, the association between smoking and CRS varies between studies ${ }^{(28,29)}$. A large epidemiological study of over 73,000 Canadians found no association between self-reported smoking and CRS; our study supports this finding (11). Similarly no association between alcohol intake and CRS was found ${ }^{(11)}$. Smoking is associated with poorer postoperative outcomes ${ }^{(30)}$.

\section{Strengths and limitations}

This study includes a varied population from across the United Kingdom. It is the largest study of CRS in the UK to date. Adoption onto the NIHR portfolio facilitated recruitment and many sites had excellent participation rates. Participants were recruited regardless of previous and subsequent management so there was no bias towards surgical or medical treatment. There should be no difference or bias regarding reporting of socioeconomic factors between controls and those with CRS.

The study design had some limitations; it was a self-reported study which predisposes to recall bias. Only those in secondary or tertiary care were included, although many of those with CRS are exclusively treated in primary care. There were large amounts of missing data for some socioeconomic parameters. If the study was redesigned, controls may have been recruited from a wider pool than just from within hospital staff or from amongst non-CRS ENT patients/relatives, to increase recruitment particularly amongst males. An online version of the questionnaire would have also produced a less labour-intensive data processing period at the end of the study.

The study did not intend and cannot provide information about prevalence of CRS in the general population.

\section{Generalisability}

Given the scope of the study incorporating a mixture of different sized academic, tertiary and secondary care sites with participants from a range of urban and rural locations around the UK, we believe the study findings are applicable to the wider population of CRS sufferers presenting to ENT departments. However, given an even larger burden of CRS patients is managed in a primary care setting, the results may not necessarily apply to the whole of the CRS-affected population.

\section{Conclusion}

Our study is the first study to assess socioeconomic influen- ces in CRS in the UK and found no socioeconomic differences between those with CRS and controls. This finding is significant in furthering our understanding of the epidemiology of CRS. We identified significant differences in health-related QoL reflecting the substantial negative effect of CRS. This increased morbidity leads to the increased health care utilisation by patients with CRS, for both nasal and non-nasal symptoms, and within both primary and secondary care. Additionally those with CRS were found to have higher respiratory and psychological co-morbidities. The disease burden associated with CRS needs to be considered in both individual patients' management and when undertaking clinical and epidemiological research into CRS, and in the context of planning future guidelines.

\section{Acknowledgements}

\section{The CRES Group:}

Chief Investigator: Mr Carl Philpott, Senior Lecturer at University of East Anglia and Honorary Consultant ENT Surgeon, James Paget University Hospital.

Miss Sally Erskine ${ }^{1}, \mathrm{Mr}$ Carl Philpott ${ }^{* \star}{ }^{*}$, Dr Allan Clark ${ }^{*}$, Miss Claire Hopkins $^{2}$, Mr Alasdair Robertson ${ }^{4}, \mathrm{Mr}_{\text {Shahzada Ahmed }}$, Mr Naveed Kara' ${ }^{12}$, Mr Sean Carrie ${ }^{11}$, Mr Vishnu Sunkaraneni ${ }^{20}$, Prof Jaydip Ray ${ }^{17}$, Mr Shahram Anari ${ }^{7}$, Mr Paul Jervis ${ }^{10}$, Miss Jaan Panesaar $^{18}$, Mr Amir Farboud ${ }^{5}$, Prof Nirmal Kumar ${ }^{3}$, Mr Russell Cathcart ${ }^{8}, \mathrm{Mr}$ Robert Almeyda ${ }^{14}$, Prof Hisham Khalil ${ }^{9}$, Mr Peter Prinsley ${ }^{13}$, Mr Nicolas Mansell ${ }^{15}$, Mr Mahmoud Salam ${ }^{16}$, Mr Jonathan Hobson ${ }^{19}$, Ms Jane Woods ${ }^{1}$, Dr Emma Coombes*

1James Paget University Hospital NHS Foundation Trust, Gorleston, ${ }^{2}$ Guys \& St Thomas' Hospital, London, ${ }^{3}$ Wrightington, Wigan \& Leigh NHS Foundation Trust, ${ }^{4}$ Southern General Hospital, Glasgow, ${ }^{5}$ Wrexham Maelor Hospital, Wales, ${ }^{6}$ University Hospitals Birmingham, ${ }^{7}$ Heart of England NHS Foundation Trust, Birmingham, ${ }^{8}$ Cumberland Infirmary, Carlisle, ${ }^{9}$ Derriford Hospital, Plymouth, ${ }^{10}$ Northampton General Hospital, ${ }^{11}$ Freeman Hospital, Newcastle, ${ }^{12}$ Sunderland Royal Infirmary, ${ }^{13}$ Norfolk \& Norwich University Hospital, ${ }^{14}$ Oxford University Hospitals, ${ }^{15}$ Royal Berkshire NHS Foundation Trust, Reading, ${ }^{16}$ The Ipswich Hospital, ${ }^{17}$ Sheffield Teaching Hospitals, ${ }^{18}$ Luton \& Dunstable Hospital, ${ }^{19}$ Warrington and Halton Hospitals NHS Foundation Trust, ${ }^{20}$ Royal Surrey County Hospital, Guildford, *Norwich Medical School, University of East Anglia, Norfolk NR4 7TJ, United Kingdom, ${ }^{\S}$ Spire Norwich Hospital.

NIHR portfolio ID: 12926; Funding: The Anthony Long Trust, the Bernice Bibby Trust.

\section{Authorship contribution}

CMP: Designed project, assisted data collection, analysis and manuscript preparation. SEE: Assisted questionnaire design, organised distribution, analysed data, prepared manuscript 
$\mathrm{CH}$ : Assisted data collection and analysis, contributed to manuscript EC: Performed geographical postcode data analysis, contributed to manuscript NK, SS, SA, MS, AF, : Assisted data collection and analysis, contributed to manuscript; AC: Data analysis including initial power calculation and planning of project, statistics and preparation of manuscript.

\section{Conflict of interest}

No authors have any conflicts of interest.

\section{References}

1. Hastan D, Fokkens WJ, Bachert C, Newson RB, Bislimovska J, Bockelbrink A, et al Chronic rhinosinusitis in Europe - an underestimated disease. A GA2LEN study. Allergy. 2011;66(9):1216-23.

2. Fokkens W, Lund V, Mullol J. EPOS 2012: European position paper on rhinosinusitis and nasal polyps. Rhinology. 2012;50(1):112.

3. Gliklich R, Metson R. The health impact of chronic sinusitis in patients seeking otolaryngologic care. Otolaryngol Head Neck Surg. 1995;113(1):(1):104-9.

4. Goetzel RZ, Hawkins K, Ozminkowski RJ, Wang $S$. The health and productivity cost burden of the "top 10" physical and mental health conditions affecting six large U.S. employers in 1999. J Occup Environ Med. 2003 Jan;45(1):5-14.

5. Office for National Statistics. Health Inequalities. Decennial Supplement No 15. London: The Stationary Office; 1997.

6. Shaw M, Dorling D, Davey Smith G. Poverty, social exclusion, and minorities. In: Marmo M, Wilkinson G, editors. Social determinants of health: Oxford University Press; 2006.

7. Davey Smith G, Blane D, Bartley MEL. Explanations for socio-economic differentials in mortality: Evidence from Britain and elsewhere. Eur J Public Health. 1994 January 1, 1994;4(2):131-44.

8. Smith DF, Boss EF. Racial/Ethnic and socioeconomic disparities in the prevalence and treatment of otitis media in children in the United States. Laryngoscope. 2010;120(11):2306-12

9. Hancox RJ, Milne BJ, Taylor DR, Greene JM Cowan JO, Flannery EM, et al. Relationship between socioeconomic status and asthma: a longitudinal cohort study. Thorax. 2004 May 1, 2004;59(5):376-80.

10. Kozyrskyj AL, Kendall GE, Jacoby P, Sly PD, Zubrick SR. Association Between Socioeconomic Status and the Development of Asthma: Analyses of Income Trajectories. Am J Public Health. 2010 2010/03/01;100(3):540-6.

11. Chen $Y$, Dales R, Lin M. The Epidemiology of Chronic Rhinosinusitis in Canadians. Laryngoscope. 2003;113(7):1199-205.

12. Greisner WA, Settipane GA. Hereditary Factor for Nasal Polyps. Allergy Asthma Proc. 1996;17(5):283-6.
13. Ahsan SF, Jumans S, Nunez DA. Chronic rhinosinusitis: a comparative study of disease occurrence in North of Scotland and Southern Caribbean otolaryngology outpatient clinics over a two month period. Scott Med J. 2004:49(4):130-3.

14. Erskine SE, Notley C, Wilson AM, Philpott CM. Managing chronic rhinosinusitis and respiratory disease: a qualitative study of triggers and interactions. J Asthma. 2015;52(6):600-5.

15. Erskine SE, Verkerk MM, Notley C, Williamson IG, Philpott CM. Chronic Rhinosinusitis: Patient Experiences of Primary and Secondary Care - A Qualitative Study. Clin Otolaryngol. 2016 Feb;41(1):8-14.

16. Bent JP, 3rd, Kuhn FA. Diagnosis of allergic fungal sinusitis. Otolaryngol Head Neck Surg. 1994 Nov;111(5):580-8.

17. Philpott CM, Javer AR, Clark A. Allergic fungal rhinosinusitis - a new staging system. Rhinology. 2011;49(3):318-23.

18. McHorney CA, Ware JE, Jr., Lu JF, Sherbourne CD. The MOS 36-item Short-Form Health Survey (SF-36): III. Tests of data quality, scaling assumptions, and reliability across diverse patient groups. Med Care. 1994 Jan;32(1):40-66

19. Hopkins C, Gillett S, Slack R, Lund VJ, Browne JP. Psychometric validity of the 22-item Sinonasal Outcome Test. Clin Otolaryngol. 2009 Oct;34(5):447-54.

20. Office for National Statistics. The National Statistics Socio-economic Classification (NS-SEC rebased on the SOC2010). 2010 [cited 2014]; Available from: http://www. ons.gov.uk/ons/guide-method/classifications/current-standard-classifications/ soc2010/soc2010-volume-3-ns-sec--rebased-on-soc2010--user-manual/index. html.

21. Department for Communities and Local Government. English Indices of Deprivation 2010. In: Government DfCaL, editor.2010.

22. Philpott C, Hopkins C, Erskine S, Kumar N, Robertson A, Farboud A, et al. The burden of revision sinonasal surgery in the UK-data from the Chronic Rhinosinusitis Epidemiology Study (CRES): a cross-sectional study. BMJ open. 2015;5(4):e006680.

23. Pilan RR, Pinna FR, Bezerra TF, Mori RL, Padua FG, Bento RF, et al. Prevalence of chronic rhinosinusitis in Sao Paulo. Rhinology. 2012:50(2):129-38.
24. Kilty SJ, McDonald JT, Johnson S, Al-Mutairi D. Socioeconomic status: a disease modifier of chronic rhinosinusitis? Rhinology. 2011;49(5):533-7.

25. Miller JD, Deal AM, McKinney KA, McClurg SW, Rodriguez KD, Thorp BD, et al. Markers of disease severity and socioeconomic factors in allergic fungal rhinosinusitis. Int Forum Allergy Rhinol. 2014;4(4):272-9.

26. Lange $B$, Holst $R$, Thilsing $T$, Baelum J, Kjeldsen A. Quality of life and associated factors in persons with Chronic Rhinosinusitis in the general population. Clin Otolaryngol. 2013;16(10):12189.

27. Wright RJ, Finn P, Contreras JP, Cohen S, Wright RO, Staudenmayer J, et al. Chronic caregiver stress and IgE expression, allergen-induced proliferation, and cytokine profiles in a birth cohort predisposed to atopy. J Allergy Clin Immunol. 2004;113(6):1051-7.

28. Hamilos DL. Chronic rhinosinusitis: Epidemiology and medical management. J Allergy Clin Immun. 2011;128(4):693-707.

29. Cerejeira R, Veloso-Teles R, Lousan N, Moura CP. Prevalence of nasaqaq4eal polyps in Northern Portugal: a cadaver endoscopic study. Rhinology. 2014;52(4):386-9.

30. Katotomichelakis $M$, Simopoulos $E$, Tripsianis G, Zhang N, Danielides G, Gouma $P$, et al. The effects of smoking on quality of life recovery after surgery for chronic rhinosinusitis. Rhinology. 2014;52(4):341-7.

\section{Sally E. Erskine}

c/o C. Philpott

Norwich Medical School

University of East Anglia

Norwich Research Park

Norwich, NR4 7TJ

United Kingdom

E-mail: sally.erskine@doctors.org.uk 